\title{
Surgical Management of Gross Mid-foot Damage
}

\author{
JOHS. G. ANDERSEN \\ Alupe Leprosy Hospital, Kenya
}

\begin{abstract}
A technique is presented for radical surgical management of gross mid-foot damage with preserved, useful plantar skin in the heel area. It is essentially a combination of pretalar amputation and calcaneo-tibial fusion with the calcaneum in 45 degrees rotation. This ensures a sound weightbearing surface and a trouble-free take-off area. This technique does not depend on sophisticated orthoses or prostheses.
\end{abstract}

\section{Introduction}

Severe damage to the mid-foot is fairly common in leprosy patients. Basically this is dependent on plantar anaesthesia. It may be a late feature of neuropathy of the foot with mid-foot collapse, ending with a boat-shaped foot with marked equinus of the calcaneum and the fore-foot in dorsiflexion. This alters the mechanics of plantar ulceration, which now predominantly takes place at the presenting plantar surface, i.e. the mid-foot. The end result is chronic ulceration with mid-foot osteomyelitis. It may also be a late result of longstanding ulceration of the fore-foot with chronic, ascending osteomyelitis of one or more metatarsal bones, usually that of the little toe, again leading to mid-foot ulceration and osteomyelitis. Valgus and varus deformities, fixed or mobile, also throw additional strain on the mid-foot with the same end result. Occasionally direct damage to the mid-foot may trigger off this unhappy sequence.

Fortunately useful sensation of the heel area is preserved in many of these feet. This, combined with the frequently found boat-shaped foot, gives rise to the rather bizarre picture of a foot with well preserved heel, occasionally in varus or valgus position, almost inevitably in equinus, a fore-foot that usually presents scars and absorptions from previous ulcerative damage, but now appears to be remarkably well preserved, and finally extensive damage to the mid-foot. In many cases this disastrous situation is not at all apparent from the dorsum, certainly not to the uneducated patient. This has been a source of some difficulties to us. It has proved comparatively easy to persuade a patient to accept an operation where "the dead and useless bones are taken out", in other words a formal fore-foot amputation or one of the modifications of transverse metatarsal head resection. It is quite another story to convince a patient that he had to have the whole, apparently normal fore-foot removed, just because of a mid-foot ulcer. I have 
even had patients suggesting that I should cut out the bad mid-foot, and then put the "good" forefoot on to the hind-foot.

Feet with gross mid-foot damage are notoriously difficult to treat. Although the basic, conservative treatment-absolute non-weightbearing, elevation of the affected limb, daily soaks in soap water, bland dressing, and judicious removal of obvious sequestra-certainly will cure the majority of these feet, it is a time consuming procedure, that effectively blocks a large number of beds. For that reason it is not a particularly cheap therapy, either. Of greater importance is the virtual certainty that these feet will re-ulcerate within a short span of time. Much effort and serious thinking have been spent on the production of orthoses that could obviate this regular re-ulceration. They are expensive, require sophisticated workshops with highly trained personnel. Even if we could persuade our patients to wear such orthoses, they simply are not available to the vast majority of them. Chances are slender that this situation will improve in the foreseeable future. Neither are there hopes that we shall see fewer people with such disabilities, when we contemplate the depressing situation of world-wide leprosy control.

In this situation it is reasonable to look for more radical therapy. One is entirely justified in abandoning the time honoured precept, "the damage to anaesthetic feet is progressive, so let us preserve whatever can be preserved". It appears logical to look for a solution that deliberately sacrifices what cannot be preserved in the long run, and to aim at serving a useful foot with a good chance of retaining it.

One very radical solution is to perform a below-knee amputation. There are 2 major drawbacks to this. First of all we condemn the patient to a definite prosthesis for life. Secondly it is hardly acceptable to most of our patients. In my view this solution is only acceptable if we are dealing with a foot where even the heel is destroyed beyond salvage.

Syme's amputation appears to offer a solution. It is comparatively easy to perform. It does not require sophisticated equipment, and can be performed adequately by doctors with less than a full orthopaedic training. However, even such modifications that prevent the classical dorsal migration of the stump pad, have serious draw-backs. The major one is that it requires a sophisticated prosthesis, which is even more difficult to make and service than a below-knee prosthesis. I entirely fail to see any advantage of this technique over a below-knee amputation.

Pirogoff's amputation is more rational. It depends on a servicable heel-pad. Its advantage is that with the frequently preserved heel sensation, we need not absolutely depend on a non-end-weightbearing prosthesis. A serious disadvantage is that the stump is too long for a comfortable prosthesis, and the leg is too short for a simple orthosis, such as an elephant boot. A valid objection to this technique is that we often find fairly extensive fibrosis round the ankle joint in these patients. This may well make impossible the required 90 degrees rotation of the posterior, osteotomized part of the calcaneum into the ankle mortice. Altogether it appears to be more difficult and less satisfactory than the technique offered in this paper.

Boyd has proposed an interesting solution. He describes a calcaneo-tibial fusion, which may be combined with a pretalar amputation. After talectomy the shaped calcaneum is fitted snugly into the denuded ankle mortice. Achillis tenotomy is essential to prevent the calcaneum from wandering. However, in the particular situation in which we are interested here, there are certain deficiencies 
in this technique. There is an inevitable shortening of the leg, though this can probably be handled with a moderate raising of the shoe. Of more importance is the fact that since the articular surfaces of the calcaneum are removed at right angles to the long axis of the leg, there is an unfortunate chance that the ulcerated/scarred part of the sole will remain within the take-off area of the foot. It is my contention that this technique as it stands is unsuitable for the particular problem with which we are dealing.

\section{Suggested Technique}

\section{INDICATIONS}

Any foot with severe damage to the mid-foot so that ulcer-free ambulation cannot be achieved.

\section{CONTRAINDICATIONS}

The only absolute contraindication that is recognized is damage to the heel to the extent that a sound weightbearing surface cannot be obtained. It should be realized that local infections and osteomyelitis do not constitute any contraindication, though they may cause the technique to be modified. Neither does the state of leprosy as such constitute any contraindication. It is well known that lepra reactions and progressive lepra as well as failure to respond properly to anti-leprosy treatment may be precipitated by such infective and necrotic conditions. General poor health with low haemoglobin readings, low plasma protein level, etc., may also be precipitated by such conditions. A radical clearance may have a very beneficial effect, indeed. On the other hand a general sepsis constitutes a relative contraindication. This must be brought under control before any surgery is attempted.

\section{TECHNIQUE}

The operation is always carried out in a bloodless field. The first incision is carried from just anterior to the tip of the medial malleolus across the ankle joint to just distal to the tip of the lateral malleolus. The second incision is carried in a long sweep across the plantar surface, outlining the longest possible plantar flap. The talo-navicular joint is opened, and the ligaments between these bones divided. Next the bones of the fore- and mid-foot are dissected off the thickest possible plantar flap until the calcaneo-cuboid joint is reached. The pre-talar bone block is removed. The ligaments on either side and the plantar surface of the talus are divided, and by putting strong equinus force on the talus it is delivered from the ankle mortice. Occasionally it is possible to remove the talus in toto. More commonly it is necessary to remove it piecemeal. This makes no difference. All joint cartilage from the ankle mortice is removed down to fresh cancellous bone. The anterior margin of the tibia is carefully removed, so that the distal surface of the tibia is flat and at right angles to the long axis of the leg. The next step is osteotomy of the calcaneum. This is a critical step. The division runs obliquely, approximately at an angle of 45 degrees with the axis of the foot. The sustentaculum tali is removed and soft tissue from the sides of the calcaneum is cleared off. After Achillis tenotomy it is now possible to rotate the osteotomized calcaneum into a snug fit in the ankle mortice. The anterior end of the calcaneum is cut down to avoid unnecessary projections beyond the anterior margin of the 


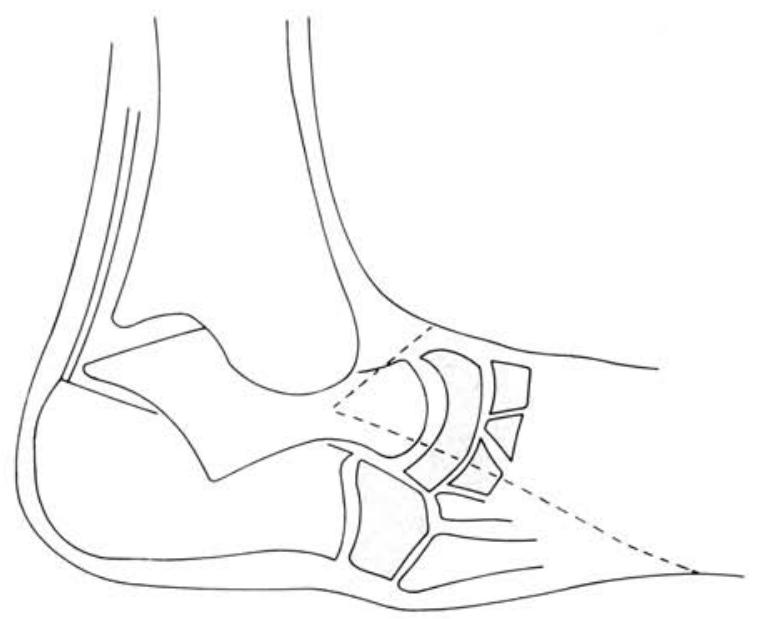

Fig. 1. The foot is shown from the tibial side. The line of incision is indicated, running from the tip of the tibial malleolus across the ankle joint to the tip of the fibular malleolus.

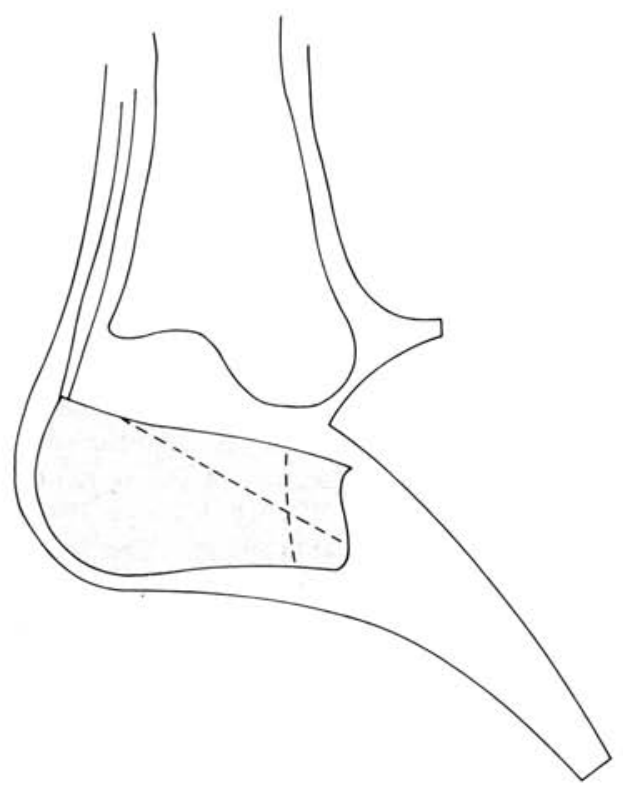

Fig. 2. After pretalar amputation and talectomy all joint cartilage from the ankle mortice is removed, and the calcaneum is osteotomized as indicated. 
tibia. The plantar flap is now trimmed to secure a snug, but not tight fit. If the flap is too loose, there a real danger of the formation of haematomata. If it is too tight, there is an equally obvious danger of edge necrosis. Before skin suture the tourniquet is completely removed and careful haemostasis is effected. Since we have to suture thin skin to thick skin, vertical mattress sutures are recommended. No attempts are made to correct the inevitable dog's ears, which might jeopardize the blood supply. Anyway, they disappear within a short time. Remaining blood and oedema is expressed, and the calcaneum is firmly pressed into the ankle mortice. A fairly voluminous dressing is applied. The whole leg up to the knee is enclosed in a thin, well-fitting plaster of Paris cast. We avoid compression clamps and rely on the compression from early ambulation. One reason is that it has been a firm objective to develop a technique that did not require excessive equipment. Another reason is that since we are operating on actually or potentially infected extremities, we consider it unwise to introduce foreign bodies with added risk of post-operative infection. The high prevalence of osteomyelitis in Africans has made us extremely careful.

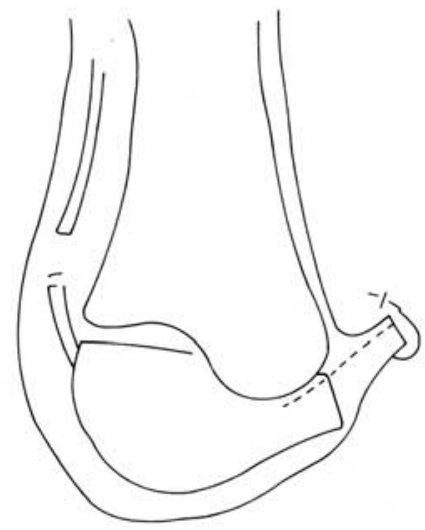

Fig. 3. After trimming of the calcaneum and Achillis tenotomy the calcaneum is rotated up and anteriorly to fit snugly into the ankle mortice. Notice that tuber calcanei is now the presenting, weightbearing surface. After trimming of the plantar flap, skin suture is carried out well away from the weightbearing and take-off surface.

\section{POST-OPERATIVE MANAGEMENT}

Elevation of the operated extremity is absolutely essential in order to avoid the formation of haematomata. This must be strictly enforced, right from the operating table, through the transport to the ward, and for at least $48 \mathrm{~h}$ after surgery. Two weeks after surgery the plaster cast is removed. If no evidence of post-operative infection or necrosis of skin edges are found, a well-fitting plaster of Paris cast, incorporating a walking device is applied, and ambulation is encouraged. At the same time the position of the heel is verified, and if necessary corrected. The walking cast is changed as required. Solid bony union might be expected from the twelfth week, although this is extremely variable. If possible, this should be confirmed radiologically. 


\section{ORTHOTICS}

After confirmation of bony union, the patient is fitted with an "elephant boot". Since there is little if any shortening of the extremity, there is no need for more raising of the foot than can be incorporated in a simple boot. There is no need for ankle braces. Since we are dealing with patients with plantar anaesthesia, that at any time might extend to the heel area, the usual precautions for such feet should be taken.

\section{COMPLICATIONS}

There are 5: healing of the suture line by secondary intention, ischaemic necrosis of the plantar flap, haematoma formation with secondary infection, continued infection from remnants of pre-existing plantar ulcers, and finally non-union.

We are dealing with at least potentially infected feet. It is therefore little wonder that a proportion of these feet do not heal by primary intention. So long as the plantar flap and the osteotomized calcaneum are secured in their proper position, this has little influence on the course, except for an unpredictable delay. It is essential in case of this complication that the foot is maintained in the proper position in a plaster of Paris cast, while weekly changes of dressing and plaster cast may be needed. Usually the whole situation quickly resolves itself, and the regime will then return to that for the uncomplicated operation.

Ischaemic necrosis of the plantar flap is, of course, a serious complication, that practically always necessitates a formal below-knee amputation. It is almost invariably caused by damage to the posterior tibial vessels during the operation. This is a technical error, and should carefully be guarded against.

Haematoma formation and secondary infection can invariably be traced to one of 2 errors, careless haemostasis or failure to secure proper post-operative elevation. Strict theatre and nursing discipline must be insisted on.

Ideally the scarred/ulcerated area of the mid-foot should be excised during the trimming of the plantar flap. This is not always possible. There is therefore at least a potential risk of continued infection. We have not found any advantage in a regime with post-operative antibiotics. If there is the slightest risk of this complication arising, it is wise to suture the 2 flaps loosely to each other. Even if such infection does develop, it rarely has any influence on the post-operative regime, other than a delay in the introduction of weightbearing.

Non-union is naturally a late complication. So far we have not experienced it. General experience would indicate that in most cases it is not a true non-union, but rather delayed union. In any case a generous trial with continued weightbearing in a walking cast should be given, before resort is taken to the drastic procedures of secondary bone grafting, etc. It is essential to maintain the calcaneum in correct position the whole time.

We have avoided introducing bone chips between the calcaneum and the ankle mortice. The reasons are the potentially infected foot on which we operate, and the high risk of haematogenous osteomyelitis in African patients.

\section{TWO-STAGE OPERATION}

Unfortunately a high number of patients present themselves with feet that are not suitable for this technique. In many cases the ideal situation is obtainable-no active ulceration, no osteomyelitis, no sequestra-if one is prepared to institute a 
time consuming regirne of conservative ulcer therapy. In many cases this is not practical. The patients may be impatient for definite treatment; and we always have the problem of bed occupancy.

Whenever it is decided to operate in spite of the ideal conditions not being met, it is wise to perform the operation in 2 stages.

The first stage is a pre-talar amputation. Since this is active osteomyelitis surgery, the incision should be left open. This, however, cannot be done, since it would jeopardize the position of the plantar flap. The compromise is to cut the plantar flap more generously and to fix it loosely with one or a few widely spaced sutures to the dorsal flap. Instead of plaster of Paris coverage, the foot is secured in a mild compression bandage over a generous padding. This is changed weekly until signs of infection have subsided. The foot is then protected in a thin plaster of Paris shell as for the definite operation. When healing is either complete or well under way, the second stage operation is embarked on. This is a calcaneo-tibial fusion after talectomy as described.

The final results of the 2-stage operation are fully comparable to those of the one-stage procedure. Naturally longer time is required before the final result is achieved.

\section{NOTES ON CHANGING OF DRESSING OR PLASTER CASTS}

In many cases a less experienced surgeon will be under considerable pressure to change plaster casts or dressing frequently, even daily. It seems to be firmly rooted in the training of nurses that any dressing should be changed soon after surgery. Many patients have an identical conviction that dressings should be changed, and wounds inspected frequently.

In the case of the one-stage procedure we are dealing with a foot with no overt pre-operative infection. There should therefore be no reason for such changes before the second week. The few indications for earlier and more frequent changes are related to the following complications:

1. Swelling of the operated extremity to the extent where there is danger of plaster constriction could indicate a haematoma. In such a case it is wise to open the plaster cast, drain a possible haematoma, and re-apply the plaster cast.

2. Post-operative infection, either from a haematoma or from inherent infection of the foot rarely emerges before the end of the first week. There will probably be some swelling of the extremity, but the picture will be dominated by a fairly copious discharge, which may seep through the plaster cast or even spill over the edge of the cast. There is usually a distinct smell, sweetish and nauseating. In these cases it is important to remove the plaster cast, ensure free drainage of the infected cavity, and maintain the foot in a well-padded compression bandage, which is then changed weekly until the situation has settled down, and a plaster cast can once more be applied. Care must be taken to maintain the osteotomized calcaneum in the correct position.

3. Ischaemic necrosis may present as a post-operative infection. More commonly it is seen as copious discharge with relatively little swelling of the extremity. One notices the characteristic sweetish nauseating smell. When this is found on opening the plaster cast, active preparations for a definite below-knee amputation must be started immediately. Obvious necrotic tissue is removed, and the extremity is treated with daily soap soaks, followed by eusol dressing. 
Eventually the raw area should be covered with a split skin graft. It is unwise to attempt a definite below-knee amputation while there still are signs of active infection in the extremity.

These conditions must be clearly distinguished from the peaceful extremity in a soaked plaster cast with a pungent, cheesy smell. This is never an indication for early change of plaster casts. It is inevitably an indication of delayed healing with some discharge, usually mixed with sloughed-off cuticle from the sole of the foot. At normal changing time a surprising amount of such "pus" can be seen. However, after simple cleaning the wound is seen to be clean and peaceful. In many cases this "pus" will be sterile.

It is notoriously difficult to rely on statements of pain from the patients. In many cases they are unable or unwilling to make the important distinction between pain and discomfort. It is also important to rely on such clinical features as raised temperature, unless of course one should have the misfortune of meeting a real, generalized sepsis, increasing WBC count or left shift of the differential count. These difficulties would appear to be connected with the fact that we are dealing with anaesthetic limbs. ESR of course is unreliable, since it may be raised anyway in many of these patients.

Changes of dressing for the first stage operation follow much the same rules, only it should be realized that here we know that we are dealing with frank infections. Still it is wise to limit the dressings to once weekly.

Experience has shown that this procedure is suitable not only for a reconstructive surgery centre, but that peripheral centres with less sophisticated experience can handle these cases competently. It has been possible to teach doctors with less than a full surgical training to perform this operation confidently, and also to select patients for it.

We have seen more than 18 months trouble-free follow-up outside the hospital.

\section{References}

Boyd, H. B. (1939). Amputation of the foot, with calcaneo-tibial arthrosesis. J. Bone J. Surg. $21,997$.

Crenshaw, A. H. (1971). (Ed.) Campbell's Operative Orthopedics, 5th edit., p. 849. C. V. Mosby Co. 